\title{
Conséquences morphologiques du traitement du conditionnel comme un temps de l'indicatif
}

\author{
Lotfi Abouda \\ Laboratoire Ligérien de Linguistique (LLL, UMR 72 70) - Université d'Orléans \\ lotfi.abouda@univ-orleans.fr
}

\begin{abstract}
Résumé. Parce qu'elle a été âprement discutée et tardive, l'admission du conditionnel au sein de l'indicatif n'a pas permis de renouveler les problématiques liées aux catégories du mode et du temps. S'appuyant sur une série d'arguments dont les plus classiques relèvent de la morphologie, cette admission n'a paradoxalement pas permis de tirer toutes les conséquences morphologiques sur l'organisation globale du système de l'indicatif. En partant d'un examen critique de l'argumentation morphologique couramment employée pour appuyer l'hypothèse de l'appartenance du conditionnel à l'indicatif, l'objectif de cette étude est de tenter une lecture morphologique qui se voudrait cohérente du paradigme temporel de l'indicatif dans son ensemble. Prise au sérieux, l'admission du conditionnel au sein de l'indicatif reviendrait à scinder la catégorie habituelle «temps » en deux catégories distinctes, dont l'une se charge de fixer l'intervalle référentiel à partir duquel se déploient les formes temporelles tandis que la seconde détermine la relation chronologique entre le procès et l'intervalle référentiel. Le conditionnel n'aurait ainsi de spécifique que le double marquage phonologique de deux morphèmes qui coexistent dans tout le paradigme de l'indicatif. Une telle vision globale, forcément schématique dans ce cadre, soulève un certain nombre de questions, dont l'opposition, sur le plan sémantique, entre le présent et l'imparfait.
\end{abstract}

\begin{abstract}
Being quite recent and heavily discussed, the acceptance of the French conditional as part of the indicative mood did not allow a renewal of the theoretical issues linked to tense and mood categories. Although based on several pieces of evidence, the most classical belonging to morphology, paradoxically this admission did not allow us to assess all the morphological consequences on the overall organization of the indicative mood. Starting from a critical examination of the morphological evidence commonly used to support the hypothesis of the conditional's belonging to the indicative mood, the aim of this study is to propose a coherent morphological reading of the entire paradigm of all the indicative tenses. When seriously considered, admitting the conditional into the indicative entails splitting the usual category of "tense" into two distinct categories. The former determines the reference interval from which tenses unfold while the latter determines the temporal relationship between the process and the reference interval. The conditional is thus only set apart by its phonological double marking of two morphemes that coexist in the whole paradigm of the indicative tenses. This global view, presented here schematically, raises a number of questions, including the semantic opposition between the present and the "imparfait".
\end{abstract}


L'admission officielle du conditionnel au sein de l'indicatif a été à la fois discutée et tardive. Figurant encore comme un mode distinct (en plus de l'indicatif, du subjonctif et de l'impératif) dans la circulaire du 25 juillet 1975, il n'apparaitra dans la liste des temps de l'indicatif, sans autres commentaires, que dans la Terminologie grammaticale de 1997 (rééditée en 1998) ${ }^{1}$. C'est sans doute à cause de cette admission tardive et quelque peu discrète que le conditionnel est encore considéré comme un mode dans la plupart des grammaires scolaires. Si, en contraste avec la situation institutionnelle et didactique, les linguistes ont été nombreux, et depuis longtemps, à considérer le conditionnel comme une forme de l'indicatif (Brunot (1905 \& 1922) Damourette \& Pichon (1911-1940) ; G. Guillaume (1929) ; R.-L. Wagner (1939)...), un examen historique précis ne parvient pas à restituer le moment qui, dans le champ linguistique, pouvait justifier ce revirement. C'est qu'il semble plus exact de parler d'évolution lente que de revirement. A l'image du Bon Usage qui, édition après édition, envisageait de plus en plus sérieusement l'idée, l'institution académique a fini par noter que, hormis la manifestation sporadique de quelques voix discordantes ${ }^{2}$, une nette majorité s'était dessinée chez les grammairiens et les linguistes en faveur du traitement du conditionnel comme une forme de l'indicatif. Ce constat semble d'autant plus adéquat qu'après avoir été longuement débattue, la question du statut catégoriel du conditionnel n'a plus été que rarement abordée dans les nombreux travaux consacrés à cette forme au cours des années 1990-2000.

Dans le champ de la morphologie, la question n'a pas non plus suscité l'intérêt des études post-structurales dans le domaine francophone. Depuis Morin (1987) et Plénat (1987), l'intérêt s'est déplacé, dans une perspective purement morphophonologique, sur les phénomènes relatifs à la défectivité et la supplétion, comme l'illustre par exemple la notion d'espace thématique ${ }^{3}$ défendue dans de nombreux travaux de Bonami et Boyé au cours des années 2000. Si ces études ont porté sur l'ensemble du système flexionnel du français, l'agencement des paradigmes y est essentiellement dicté par les variations de forme qui affectent le radical verbal, et ne cherche ni à s'appuyer sur des hypothèses grammaticales existantes, ni à contribuer à en formuler de nouvelles. On trouvera dans Aronoff (2012) un exemple, particulièrement parlant, de ce type de démarche où on s'autorise, au gré de l'allomorphie radicale, à regrouper, indistinctement, des affixes modaux et temporels, ou, inversement mais tout aussi indistinctement, à éclater l'unité d'un paradigme temporel. Il est clair que dans des cadres de ce type la question du statut catégoriel du conditionnel ne peut pas être jugée pertinente.

Cette situation justifie que l'on reprenne ici cette question, non pas pour la reposer, mais pour essayer de tirer les conséquences morphologiques d'un tel traitement sur le système de l'indicatif dans sa globalité. Vaste question qui dépasse de beaucoup le cadre offert ici : il ne sera donc question que d'une esquisse qui laissera ouvertes nombre d'interrogations, et évitera, faute de place, la discussion des thèses morphophonologiques évoquées ci-dessus.

Après une première partie, où il sera question d'interroger la validité de l'argumentation morphologique appelée à l'appui du traitement du conditionnel comme forme de l'indicatif, nous proposerons, dans une deuxième partie, une vision globale $\mathrm{du}$ mode indicatif qui s'appuie sur les données morphologiques retenues auparavant, avant d'évoquer dans un troisième temps les principales conséquences sémantiques d'un tel traitement. 


\section{Morphologie du conditionnel}

Les arguments avancés en faveur du traitement du conditionnel comme temps de l'indicatif sont nombreux et variés, allant de la diachronie jusqu'à la syntaxe ${ }^{4}$, en passant par la morphologie. Cette dernière occupe une place de choix dans nombre de travaux. Touratier (1996:38) la résume ainsi :

Il est difficile de ne pas retrouver dans le conditionnel d'une part la marque de l'imparfait et d'autre part la marque /R/ du futur, comme le montrent les deux paires minimales :

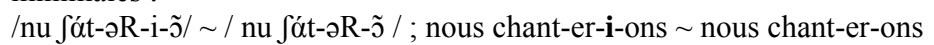

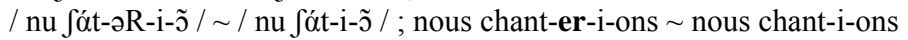

Ceci veut dire qu'au point de vue morphologique, le conditionnel dit présent a tout l'air d'être un futur imparfait et donc d'appartenir aux temps de l'indicatif. Car si le futur est un temps de l'indicatif et l'imparfait un autre temps de l'indicatif, on ne voit pas comment la combinaison de ces deux temps de l'indicatif pourrait ne pas appartenir aussi au mode indicatif.

Si cette approche par ajout, qui consiste à voir dans la marque du conditionnel la combinaison des morphèmes de l'imparfait et du futur, est extrêmement répandue à la fois dans les grammaires et chez les spécialistes des temps ${ }^{5}$, elle n'est pas sans poser quelques difficultés.

D'abord, l'unanimité constatée cesse dès que se pose la question du radical verbal auquel s'attache le morphème du conditionnel. Si la totalité des travaux postulent à juste titre un radical commun au futur et au conditionnel, ils divergent sensiblement sur la nature du radical en question : s'agit-il de l'infinitif du verbe ou d'un radical commun à tous les temps de l'indicatif auquel s'attache, au futur et au conditionnel, un $/ \mathrm{R} /$ spécifique et distinct de celui de l'infinitif ? S'agissant d'une question complexe et très débattue ${ }^{6}$, on se contentera ici d'admettre que le $/ \mathrm{R} /$ commun au futur et au conditionnel est un morphème flexionnel distinct de celui de l'infinitif, en évoquant brièvement les arguments suivants :

1. D’abord, ainsi que le note Camussi-Ni (2006 : 47),

Conserver le $\mathrm{R}$ dans la base, c'est créer un allomorphisme de la base qui ne se trouverait que dans deux variantes : le conditionnel présent et le futur. C'est affirmer la corrélation entre cet allomorphe $+\mathrm{R}$ et ces deux temps, ce qui équivaut à dire que le matériau R participe de la dénotation du futur et du conditionnel présent. Puisqu'il est pertinent d'isoler un suffixe dans l'infinitif, puisque le suffixe $\mathrm{R}$ du conditionnel est porteur d'un sème spécifique en français moderne comme le montre l'opposition du conditionnel et de l'imparfait, on ne voit pas l'intérêt qu'on peut avoir à éviter de le découper. On court plutôt le risque alors d'opacifier l'analyse du futur et du conditionnel.

2. Sauf que la commutation n'est pas à appliquer mécaniquement sans tenir compte du contexte syntaxique, qui permet de distinguer nettement la distribution de l'infinitif de celle de l'indicatif, dont le futur et le conditionnel. Autrement dit, la commutation ne parle que lorsque, au lieu d'être appliquée à des formes verbales isolées, s'applique à des formes comparables qui apparaissent dans les mêmes contextes syntaxiques.

3. Sur le plan purement morphophonologique, si nous sommes d'accord avec Bonami (2014) pour affirmer qu'aucun argument empirique ne permet de trancher la question, il nous semble moins coûteux d'identifier pour le futur et le conditionnel un morphème $/ \mathrm{R} /$ distinct de l'infinitif. $\mathrm{Si}$ l'affirmation nous paraît aller de soi pour les verbes $\mathrm{du} 1^{\mathrm{er}}$ groupe, où l'identité entre le $\mathrm{R} d u$ futur/conditionnel et celui de l'infinitif n'est qu'orthographique, et non phonologique (le -er de l'infinitif est [е] et non [әк] : danser [dãse] mais danserai [dãsəье]), elle nous semble tout aussi pertinente pour les verbes des $2^{\mathrm{e}}$ et $3^{\mathrm{e}}$ groupes, où tout traitement alternatif, en plus de rompre le paradigme des temps, ne parvient pas véritablement à réduire l'allomorphie ${ }^{7}$ : que l'on compare le radical verbal de l'infinitif et du futur (et des autres temps de l'indicatif!) pour des verbes comme courir, tenir, pleuvoir, voir, savoir...

Ensuite, l'argumentation compositionnelle pourrait sembler paradoxale, parce que par le simple fait de voir dans la marque du conditionnel la combinaison de deux morphèmes temporels, elle l'exclut du 
paradigme disjonctif de l'indicatif, dans lequel sont censées s'opposer directement les 5 formes temporelles simples (et leurs correspondants complexes), i.e. présent (PR), futur (FUT), passé simple (PS), imparfait (IMP)... et conditionnel (COND). Autrement dit, l'hypothèse de la compositionnalité du conditionnel, si elle devait être prise au sérieux, aurait pour conséquence paradoxale de ne plus admettre cette forme dans le même paradigme temporel que l'imparfait et le futur, et, donc, de porter un coup à l'analyse du conditionnel comme un temps de l'indicatif.

Enfin, ce type d'argumentation pourrait même paraitre défectueux, et interroge la notion elle-même de morphème. Considérer la marque du conditionnel comme un morphème tout en y voyant la combinaison de deux autres morphèmes, ceux de l'IMP et du Fut, reviendrait à attribuer à cette forme deux structures internes concurrentes que l'on peut représenter ici sous la forme simplifiée suivante (faisant notamment abstraction du Mode et de l'Aspect ${ }^{8}$ et de divers types d'allomorphie), en choisissant comme forme de référence un verbe du $1^{\text {er }}$ groupe à la $1^{\text {ère }}$ personne du pluriel :

Tableau 1 Deux structures morphologiques concurrentes
A. Radical $+R+\mathrm{i}+\mathrm{PN}$
B. Radical + temps $+\mathrm{PN}^{9}$

$\begin{array}{ll}\varnothing & \text { PR } \\ \mathrm{a} & \text { PS } \\ \mathrm{R} & \text { FUT } \\ \mathrm{i} & \text { IMP } \\ \mathrm{Ri} & \text { COND }\end{array}$

L'abandon de l'une ou l'autre des deux lectures (A)/(B) semble incontournable. Généralement, c'est le traitement (A) et le(s) schéma(s) morphologique(s) qu'il implique qui se trouvent, sinon abandonnés, du moins largement sous-exploités : mise en avant quand il est question du conditionnel ou de son statut catégoriel, la compositionnalité de cette forme n'est que rarement réinvestie dans l'analyse du système de l'indicatif dans son ensemble.

Les raisons de cette sous-exploitation sont nombreuses. D'abord, elle implique un bouleversement non seulement de la manière de percevoir le système temporel de l'indicatif, mais aussi de la catégorie habituelle du temps. A quelques exceptions notables près, notamment Damourette \& Pichon et Coseriu, le prix, une remise en cause totale de l'organisation globale du système de l'indicatif, était jugé trop exorbitant par la plupart des grammairiens et linguistes. Ensuite, l'adoption du schéma (A) donne aux morphèmes zéros un rôle central dans l'organisation des formes temporelles. Or, un principe tacite existe, qui consiste à se méfier du zéro. Et même quand on est obligé d'admettre le zéro, c'est souvent à titre provisoire, en adoptant l'attitude, décrite par Gleason (1969), qui consiste à éliminer de la description finale les zéros introduits provisoirement au cours de l'analyse ${ }^{10}$. Camussi-Ni, discutant les travaux classiques en morphologie structurale, écrit $(2006: 61)$ :

\footnotetext{
C'est parce que la marque est assimilée à du matériau et non pas à un rapport entre matériaux qu'il paraît difficilement concevable que le matériau zéro soit une marque. Or, précisément le zéro n'est pas une marque, pas plus que ne l'est le matériau auquel il s'oppose, c'est l'opposition significative entre la présence et l'absence de matériau qui constitue la marque.
}

Ce sont des principes généralement admis, notamment quand il est question de décrire des langues « exotiques ». Leur adoption rencontre une certaine résistance lorsqu'on cherche à les appliquer à des langues à forte tradition écrite.

En bref, l'argumentation morphologique exposée ci-dessus ne pourrait être maintenue ${ }^{11}$ que si elle est réellement prise au sérieux et que toutes ses conséquences en soient tirées. 
Pour nécessaire, l'adoption du schéma (A) n'est pas suffisante pour assoir sur des bases morphologiques solides le traitement du conditionnel comme un temps de l'indicatif. A supposer que soient résolus tous les problèmes, nombreux et complexes, qu'un tel schéma implique, son adoption n'écarte pas longtemps les traitements concurrents.

Concrètement, le schéma (A) peut être généralisé comme sous (1) :

$$
\text { (1) Radical }+\mathrm{X}+\mathrm{Y}+\mathrm{PN}
$$

Notre lecture, qui sera développée ci-dessous, n’est pas la seule possible. On pourrait en effet penser que $\mathrm{X}$ est une catégorie de Mode, et non de Temps. Dès lors, rien n'empêche de considérer ${ }^{12}$, comme le fait par exemple H. Yvon (1952) $)^{13}$ ou encore plus récemment Picabia (1999), la marque - $R$ - sous X, commune au futur et au conditionnel, comme le morphème d'un Mode qu'on peut appeler Suppositif. Le système se déclinerait alors de la manière abrégée et simplifiée suivante :

Tableau 2 : Système d'Yvon (1952)

\begin{tabular}{|c|c|c|c|c|}
\hline \multirow{2}{*}{ Radical } & \multicolumn{2}{|c|}{ Mode } & \multicolumn{2}{c|}{ Temps } \\
\hline \multirow{3}{*}{ indicatif } & $\varnothing$ & $\varnothing$ & PR \\
\cline { 3 - 5 } & \multirow{2}{*}{ suppositif } & & $-\varepsilon-$ & IMP \\
\cline { 3 - 5 } & & & $\varnothing$ & FUT \\
\cline { 3 - 5 } & & & $-\varepsilon-$ & COND \\
\hline
\end{tabular}

Le traitement d'Yvon est d'autant plus envisageable que le statut modal ou temporel du futur lui-même a toujours fait débat..

Ce rapide tour d'horizon avait pour objectif de montrer que l'argumentation morphologique classique, aussi répandue soit-elle, ne résiste pas à un examen détaillé des faits et ne permet pas de montrer d'une manière indiscutable l'appartenance du conditionnel à l'indicatif.

\section{Morphologie de l'indicatif}

Si les considérations strictement morphologiques étaient jugées ci-dessus insuffisantes pour déterminer le statut catégoriel du conditionnel, l'hypothèse de son appartenance à l'indicatif, adoptée sur la base d'un ensemble d'autres arguments, a des conséquences morphologiques sur tout le système temporel, qu'il est nécessaire d'examiner. Ce chemin, s'il n'est pas habituel - les traitements généralistes classiques commencent systématiquement par le présent pour finir par le conditionnel - nous semble à la fois plus riche et, sans doute, paradoxalement plus simple.

Ainsi qu'il a été affirmé ci-dessus, les deux structures concurrentes (A) et (B) esquissées dans le tableau 1 , ne peuvent pas être simultanément attribuées au conditionnel. Nous appuyant sur de nombreux travaux qui soulignent les liens du conditionnel avec l'imparfait et le futur, nous nous proposons d'explorer dans ce cadre la lecture (A), reproduite ici par commodité :

$$
\text { (2) Radical }+\mathrm{R}+\mathrm{i}+\mathrm{PN}
$$

On peut naturellement penser que la structure morphologique (et sémantique) du conditionnel est singulière : de très nombreux travaux, à la fois en morphologie ${ }^{15}$ et en sémantique ${ }^{16}$, lui accordent un traitement spécifique. Mais il est également possible de penser que cette singularité est douteuse, et que la structure du conditionnel n'a de spécifique que le double marquage phonologique de deux morphèmes qui coexistent dans tout le paradigme de l'indicatif. Cela revient à voir dans la catégorie traditionnelle 
« temps » une catégorie générique, amalgamant systématiquement deux catégories distinctes, selon le schéma morphologique (simplifié, où l'on ne tient compte ni du Mode ni de l'Aspect) :

(3) Radical $+\mathrm{X}+\mathrm{Y}+\mathrm{PN}$

A partir de ce schéma global, on peut organiser les formes temporelles de l'indicatif de différentes manières $^{17}$. Nous nous proposons de décliner ici ce schéma morphologique simplifié de la manière suivante :

Tableau 3 : Morphèmes temporels de l'indicatif

\begin{tabular}{|c|c|c|c|}
\hline \multirow{6}{*}{ 点 } & $\mathbf{X}$ & $\mathbf{Y}$ & Temps \\
\hline & $\varnothing$ & $\varnothing$ & PR \\
\hline & $\varnothing$ & $\mathrm{i}$ & IMP \\
\hline & $\mathrm{a}$ & $\varnothing$ & PS \\
\hline & $\mathrm{R}$ & $\varnothing$ & FUT \\
\hline & $\mathrm{R}$ & $\mathrm{i}$ & COND \\
\hline
\end{tabular}

où on note la présence de deux paradigmes parallèles, appelés provisoirement $\mathrm{X}$ et $\mathrm{Y}$, nés de la fission de la catégorie habituelle « temps ».

Le paradigme $\mathrm{X}$ oppose trois morphèmes :

$\varnothing$ : morphème de concomitance : PR ou IMP, selon la valeur de $\mathrm{Y}$

$\mathrm{a}$ : morphème d'antécédence : PS (qui n'apparaît que si $\mathrm{Y}=\varnothing$ )

$\mathrm{R}$ : morphème de prospection : FUT ou COND, selon la valeur de $\mathrm{Y}$.

$\mathrm{Si}$, servant à situer chronologiquement les procès par rapport à un repère référentiel, la catégorie $\mathrm{X}$ se présente comme une catégorie strictement temporelle, la détermination de la catégorie Y peut en revanche s'avérer plus délicate.

Opposant un morphème $\varnothing$ au morphème /i/, la catégorie Y permet de répartir les temps de l'indicatif en deux sous-groupes distincts, comme on peut le visualiser dans le tableau suivant:

Tableau 4 : Deux groupes de formes temporelles

\begin{tabular}{|c|c|c|c|}
\hline \multirow{6}{*}{ 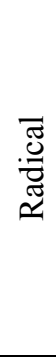 } & $\mathrm{X}$ & $\mathbf{Y}$ & Temps \\
\hline & $\varnothing$ & \multirow{3}{*}{$\varnothing$} & PR \\
\hline & $\mathrm{a}$ & & PS \\
\hline & $\mathrm{R}$ & & FUT \\
\hline & $\varnothing$ & \multirow{2}{*}{$\mathrm{i}$} & IMP \\
\hline & $\mathrm{R}$ & & COND \\
\hline
\end{tabular}

(i) le premier groupe $(\mathrm{Y}=\varnothing)$ a pour centre le présent, et contient en plus de lui, selon la valeur de X, le futur et le PS (et leurs correspondants composés).

(ii) Le second groupe $(\mathrm{Y}=\mathrm{i})$ comporte l'IMP et le COND (et leurs formes temporelles composées correspondantes). 
L'identification de la catégorie Y exige d'identifier au préalable ce qui oppose les deux groupes de formes temporelles, et d'abord leurs deux repères-origines respectifs, correspondant au PR et à l'IMP.

L'opposition PR/IMP, et plus généralement le point référentiel à partir duquel s'envisage l'IMP, est l'une des questions les plus traitées dans le champ de la temporalité. Si c'est loin d'être la seule piste explorée, l'opposition PR-IMP a souvent été décrite en termes temporels. Or, si on envisage sérieusement le schéma (3), voir dans Y une seconde catégorie temporelle reviendrait de fait à la faire disparaître : les deux paradigmes parallèles finiraient par fusionner à nouveau et les zéros n'auraient été qu'un outil avancé provisoirement au cours de l'analyse avant de disparaitre... Bref, on reviendrait au schéma habituel, où il n'y aurait qu'une seule catégorie temporelle, et où on peut voir chacune des formes simples de l'indicatif comme une forme homogène, sauf le conditionnel, matériau oblige, dans lequel on va continuer à voir tout de même une forme composite et singulière. Or, nous l'avons vu, ce traitement singulier va à l'encontre de l'intégration pleine du conditionnel au sein de l'indicatif.

\section{Une vision de l'indicatif}

\subsection{Arguments pour une bi-partition}

L'hypothèse de l'existence à l'indicatif de deux groupes de formes temporelles s'appuie ici sur des considérations morphologiques : c'est l'une des lectures rendues possibles par les jeux paradigmatiques. Mais l'idée elle-même de cette bipartition (ou de bipartitions proches) n'est pas nouvelle. On peut même penser que, remontant à plus de trois siècles, elle est bien ancrée dans la tradition linguistique, dès lors qu'on accepte de la détecter sous des appellations et des formes variées : temps simples/temps composés (dans le sens) chez Arnauld \& Lancelot $(1660)^{18}$, temps absolus/temps relatifs (Girard (1747), Brunot (1922)...) ; temps noncaux / temps toncaux (Damourette \& Pichon (1911-1940); temps actuels/temps inactuels (Coseriu (1980)...) ; temps déictiques/temps anaphoriques (Maingueneau (1981), Tasmowski (1985), Molendijk (1996), Kleiber (1993)...), premier sous-système/second sous-système (Wilmet $(1997) \ldots{ }^{19}$

Naturellement, toutes ces bipartitions, avancées dans les cadres théoriques les plus divers, ne sont pas équivalentes, et comportent même des différences majeures. Par exemple, si certains auteurs classent des tiroirs, certains autres classent des emplois, en s'appuyant le plus souvent sur des exemples fabriqués. L'extension elle-même de chaque groupe, s'appuyant sur des définitions différentes plus ou moins explicites de la nature de l'opposition, peut varier d'une bipartition à une autre ${ }^{20}$. Enfin, ces bipartitions ont souvent laissé place à des tripartitions, comme on peut le voir avec l'apparition de la classe des anadéictiques ou encore la classe de la temporalité absolue-relative proposée par Comrie (1985) dans une perspective de linguistique générale ${ }^{21}$.

Il n'empêche que l'idée qu'il existe au sein de l'indicatif deux sous-systèmes parallèles dont l'un est organisé autour du présent et l'autre autour de l'imparfait semble être une constante dans un champ grammatical et linguistique, connu par ailleurs pour sa grande diversité. Ce constat historique et épistémologique a déjà été souligné par Wilmet (1996:200) :

\footnotetext{
Les grammairiens ont longtemps vu dans l'imparfait français un "présent du passé" (Maurice Grevisse jusqu'à la septième édition du Bon usage, en 1961) ou un "deuxième présent" (Weber 1954), ou un "passé du présent" (la majorité des francistes espagnols et italiens). Ces formulations maladroites traquent une ressemblance intuitivement perçue de l'imparfait et du présent.
}

Ne résistons pas davantage à la tentation de citer l'Abbé Girard (1647 : t.2, p. 25) qui présente sa vision de la bipartition de la manière suivante :

On pourroit en faire la distinction par les simples épithètes numérales de premier \& second : mais pour les mieux caractériser je les distingue par celles d'absolu \& relatif. Lorsqu'ils représentent le temps de l'événement par la seule comparaison avec celui où l'on parle ils sont TEMPS ABSOLUS ; lorsqu'ils le représentent par une double 
comparaison, non seulement avec le temps de la parole, mais avec celui de quelque autre événement, ils sont TEMPS RELATIFS.

Sans aller jusqu'à dire, à l'instar de Vetters (1996: 45), que cette définition «n'a rien perdu de son actualité et que les définitions actuelles reviennent exactement au même », il nous semble en effet que, malgré une évolution lente, une traque collective s'est engagée depuis longtemps pour identifier ce différentiel insaisissable entre deux paradigmes temporels, et partant entre leurs deux centres respectifs, PR vs IMP.

\subsection{De l'opposition PR/IMP}

L'opposition entre le présent et l'imparfait ayant fait l'objet d'un nombre considérable d'études linguistiques, il serait irréaliste de penser que nous pourrions ici, au détour d'une argumentation, aborder exhaustivement un domaine très largement débattu et très complexe. Nous nous contenterons donc de faire un certain nombre d'observations, en privilégiant la présentation des analyses qui, quel que soit leur cadre théorique, semblent appuyer la vision défendue ici.

D'abord, il est important de rappeler, suite à Fournier (1986), que c'est bien l'héritage latin qui explique le traitement de l'imparfait comme un temps du passé. "Jusqu'à Port-Royal, écrit-elle (1986 : 15), la problématique de l'imparfait frappe par sa stabilité, évidemment due à l'héritage latin: pour les grammairiens, j'aimais, c'est amabam, et ils se félicitent de cette équivalence». Centrées sur la morphologie et l'étude des distributions dans une visée essentiellement didactique, les "petites grammaires d'usage », dont le représentant le plus illustre sera Maupas, auraient pu, grâce à l'attention qu'elles portaient aux emplois, y compris «non-canoniques », et aux propriétés aspectuelles de la forme, nuancer plus fermement la caractéristique exclusivement chronologique attribuée à l'imparfait. Mais PortRoyal, s'attachant à proposer une systématique temporelle "logique », et même dans le cadre de la bipartition temps absolus/temps relatifs, écartera toute considération aspectuelle et négligera les emplois, avec les conséquences que l'on sait ${ }^{22}$. On ne peut ne pas faire le lien entre la domination de la lecture chronologique de l'imparfait et le triomphe institutionnel et symbolique de Port-Royal.

S'il est vrai aujourd'hui que la plupart des approches proposent pour l'imparfait un traitement aspectuotemporel, il a toujours existé, avancés dans les cadres les plus divers, des traitements qui refusent de voir en l'IMP un temps (exclusivement) du passé.

Ainsi, pour Damourette \& Pichon, ce temps, qui « est loin d'exprimer forcément un passé » $(\S 1709)^{23}$, signifie toujours «l'abandon du repérage par rapport au moi-ici-maintenant» $(\S 1730: 226)^{24}$. Ils attribuent ainsi à l'IMP une valeur sémantique négative et vague de type non-présent, ou non-actuel, une sorte de non-[Moi-Ici-Maintenant].

On trouvera chez Coseriu (1980) une analyse similaire qu'il défendra dans une approche romanistique. Distinguant deux dimensions temporelles, i.e. le plan et la perspective, il définit la première $(1980: 19)$ comme

la dimension au moyen de laquelle certaines actions verbales sont placées directement par rapport à l'acte de la parole sur une ligne de réalité non-diminuée, tandis que d'autres actions sont placées comme sur une arrière-ligne (conditions, circonstances, etc. des actions "actuelles") et présentées comme plus ou moins diminuées dans leur effectivité.

Cette distinction, qui constitue d'après Coseriu le système de base de toutes les langues romanes, permet d'opposer deux sous-systèmes temporels : l'actuel, centré sur le PR ; et l'inactuel, ayant pour centre l'imparfait, qui, selon l'auteur (ibid.) «n'est pas à proprement parler un temps du passé ».

Solidement ancrée dans la tradition linguistique, cette hypothèse reste défendue dans de très nombreuses études linguistiques actuelles, où elle joue souvent un rôle explicatif central. Leur peu de visibilité et d'impact, en tout cas en dehors du champ de la temporalité, s'explique à nos yeux à la fois par la résistance, soulignée ci-dessus, de l'institution grammaticale, et, surtout, par leur dispersion dans des 
cadres théoriques étanches qui, se livrant à des polémiques théoriques sans fin, ne permettaient pas toujours aisément de voir la similitude entre les traitements.

Pour ne donner qu'un exemple illustrant l'actualité de cette hypothèse ${ }^{25}$, on peut évoquer les études directement inspirées du modèle théorique de Culioli et de l'analyse esquissée dans son article de 1980. Afin de rendre compte des propriétés de l'imparfait, l'auteur y propose l'établissement d'un repère translaté, défini par sa relation de différenciation par rapport au repère d'origine. Reprenant le même cadre théorique, Lebaud (1993) attribuera à l'imparfait, outre la propriété négative de ne pas permettre de singulariser une classe d'instants (seuls les temps calculés par rapport au moment de l'énonciation permettent de singulariser une classe d'instants), la propriété positive de marquer un changement de repère, c'est-à-dire la construction d'un nouveau repère à partir d'un repère-origine. De nombreuses études développeront ces vues au cours des années 1990 et 2000.

En second lieu, on remarquera que si l'imparfait est, et de loin, la forme temporelle la plus polysémique ${ }^{26}$, le processus, vu ci-dessus, qui a consisté à neutraliser l'extrême variation dont il est porteur, a débuté dès le $\mathrm{XVII}^{\mathrm{e}}$ siècle pour le faire entrer dans la seule case que le système logique classique, sous l'influence de l'héritage gréco-latin, pouvait lui laisser, celle d'un temps du passé. Il se trouve que les seuls auteurs qui, comme Damourette \& Pichon, remettaient en cause le statut exclusivement temporel de l'imparfait étaient les mêmes qui acceptaient l'extrême richesse des emplois et la grande variété des usages. En face, la décision de traiter l'imparfait comme (d'abord) un temps du passé n'était compatible avec la prise en compte des emplois « déviants » ou «non-canoniques » qu'au prix de leur marginalisation. Ces derniers étaient alors vus comme secondaires, issus de cette valeur première par des opérations de dérivation dont Confais dira (1990 : 53) qu'elles « se font au coup par coup au moyen d'analyses ad hoc qui nécessitent un certain talent de la part du linguiste, car elles s'avèrent parfois très acrobatiques ».

A l'appui du traitement de l'IMP comme temps de passé, il n'y a pas uniquement l'héritage d'un champ, mais des arguments plus linguistiques, comme celui, présenté souvent comme décisif, qui consiste à affirmer qu'un énoncé à l'IMP, hors contexte, situe forcément le procès dans le passé, comme dans l'exemple suivant :

\section{(4) Il pleuvait}

La validité d'un tel argument est discutable. Objet sémantique, le temps ne s'observe pas au niveau de la phrase, car l'exemple (4) représente bien une phrase, et non un énoncé ${ }^{27}$. Il est en effet admis dans la quasi-totalité des courants théoriques que c'est même le contexte qui différencie l'énoncé, seul observable, de la phrase, objet construit. En bref, un énoncé hors contexte n'existe pas et l'objet temps ne peut pas être appréhendé comme on appréhenderait un objet syntaxique.

Enfin, il convient de rappeler que s'il est vrai, ainsi que nous le verrons ci-dessous, que les modèles qui voient dans l'imparfait une forme verbale de décrochage par rapport à la situation d'énonciation butent paradoxalement sur l'emploi chronologique, il existe toute une série d'emplois qui « paraissent battre en brèche les approches aspecto-temporelles, offrant à ceux qui ne placent pas la référence temporelle au centre de leurs analyses des exemples de choix pour défendre leurs thèses » (De Saussure \& Sthioul (2005 : 103). On peut, pour fixer les idées, illustrer ces classes d'emplois par les exemples empruntés suivants, qui sont loin d'être représentatifs de tous les cas identifiables ou identifiés, et de tous les souscas que l'on peut rencontrer dans une classe donnée d'emplois :

\section{(5) Imparfait 'hypothétique'}

(a) Il songeait : - Si je pouvais tuer tous ceux qu'elle a aimés ! (A. France cité par Damourette \& Pichon) ${ }^{28}$

(b) Et dire que sans vous, j'étais tranquillement dans la cuisine en ce moment, ou à me chauffer au soleil dans le jardin en attendant la cloche du déjeuner. (M. Maeterlinck, cité par Damourette \& Pichon)

(c) Mon paquet! eh, dis donc, les bonnes femmes elles dansaient à poil ce soir, elles n'avaient pas de maillot! (cité par Damourette \& Pichon) ${ }^{29}$

(6) Imparfait 'hypocoristique' 
Dans un phylactère de la bande dessinée Drame de la jalousie (Brétécher, Les Frustrés, 5), un homme, qui garde les enfants de son amie en son absence, tente de consoler le petit garçon d'un gros chagrin en le prenant dans ses bras et dit :

- Ch'est un pauvre bébé cha ... il avait le cœur brijé ... (Barceló \& Bres, $2006: 62)$

(7) Imparfait de 'décrochage subjectif' (Lebaud) /ground décalé (De Mulder)

Zut ! Dimanche prochain, il y avait un petit marathon sympa à Reims! (Lebaud)

(8) Imparfait 'atténuatif', 'de politesse' ou 'de discrétion'

(a) Ecoute, Caroline, avant que tu me quittes, je voulais te demander quelque chose.

(F. Mauriac, cité par Damourette \& Pichon)

(b) Je venais te dire bonsoir. (A. Gide).

(9) Imparfait 'préludique' :

Après avoir choisi de jouer à l'« école des grands » (A est la maîtresse et B l'élève), A explique la situation de jeu à $\mathrm{B}$.

A. mais tu prends pas le bébé parce que t- $\mathrm{t}$ - en fait $\mathrm{i}$ i faut $\mathrm{i}$ faut pas que ta maman vienne en fait et puis et puis ton papa ni et be et be i faut y aller tout seul d'accord?

B. mais j'comprends plus rien moi hein !

A. ba au début tu savais pas //

B. au début je savais pas qu'est-ce que je savais pas au début?

A. au début tu savais que / queum qu'en fait que moi j'étais // euh // le fait que moi je suis la maîtresse et toi tu es la grande sœur / mais tu apportes pas le bébé et puis moi je // d'accord mais tu apportes rien (Patard, 2007)

Sans minimiser la pertinence et l'apport des études adverses, il nous semble que la plupart des modèles non-temporalistes parviennent mieux et à moindre coût ${ }^{30}$ à traiter les emplois dits modaux.

Enfin, reconnaissons tout de même que c'est face à l'emploi chronologique de l'imparfait, notamment en combinaison avec le PS, que les approches non-temporalistes rencontrent le plus de difficultés. Examinons, pour fixer les idées, l'exemple suivant de Zola, cité par De Mulder (2012), qu'il commentera à la suite :

(10) Il lui empoigna la tête, il la cogna contre un pied de la table. Elle se débattait, il la tira par les cheveux, au travers de la pièce, bousculant les chaises. Chaque fois qu'elle faisait un effort pour se redresser, il la rejetait sur le carreau d'un coup de poing. [...] La table poussée, faillit renverser le poêle. Des cheveux et du sang restèrent à un angle du buffet. Quand ils reprirent haleine, hébétés, gonflés de cette horreur, las de frapper et d'être frappée, ils étaient revenus près du lit... (La Bête humaine)

De Mulder affirme $(2012$ : 105) à juste titre qu'il n'est pas possible de soutenir que l'imparfait dans cet extrait exprime un déplacement du point de vue, comme c'est le cas des exemples suivants, qu'il emprunte respectivement à Arrivé, Gadet \& Galmiche $(1986: 309)$ et Wilmet $(1996: 206)$ :

(11)(a) Dommage, il était beau ce vase. (À propos d'un vase que l'on décide finalement de ne pas acheter)

(b) C'est bien vous qui parliez lors de la prochaine réunion ?

En effet, il paraît indiscutable que les procès à l'IMP dans la séquence (10) soient vus du même point de vue que ceux au PS. Les traitements inspirés du modèle de Langacker selon lesquels l'IMP désignerait une réalité non immédiate se trouveraient ainsi en difficulté. Liées au sein d'une même scène narrative, les deux formes temporelles exigeraient un traitement similaire que le modèle, sous peine d'invalidation, ne peut offrir en attribuant à l'IMP une propriété qu'il refuserait au PS.

En réalité, cette critique peut souvent être émise à l'encontre de la plupart des approches nontemporalistes dans ce type de contexte narratif, où la trame narrative fonctionne grâce à une répartition de 
rôles au sein du couple IMP-PS. Des solutions existent mais risquent d'être invalidées dans d'autres contextes...

\title{
3.3 Portrait-robot d'une proposition
}

L'imparfait peut être considéré comme une forme verbale non saturée. Cette non-saturation, qui n'est pas sans rappeler le traitement anaphorique ni la notion d'incomplétude (Le Goffic, 1995), provient d'une autre caractéristique, soulignée dans beaucoup de travaux vus ci-dessus, qui voit dans l'imparfait un nonprésent. C'est parce qu'elle a perdu son attache par rapport à la situation énonciative $\left(\mathrm{t}_{0}\right.$, actualité, nunc, repère-origine... peu importe la terminologie ici), que cette forme cherchera à se fixer au premier repère référentiel disponible, quelle qu'en soit la nature. Et dans les énoncés réels, il y a toujours un repère disponible, même s'il faut quelquefois aller le chercher bien loin.

Si cette approche, écartant ce que Confais (1990) appelle la thèse du signifié fondamental ${ }^{31}$, évite les procédures de dérivation (ou de conversion, re-catégorisation, coercion, etc. ${ }^{32}$ ), elle reste sous la menace d'une seconde objection que Gosselin (2010 : 114) adresse à l'égard de la démarche dérivationnelle mais qui ne perd pas totalement sa pertinence ici, celle de

\begin{abstract}
vider presque intégralement de leur signification [les marqueurs], ou [de] ne pouvoir décrire cette signification de base que de façon purement négative (par ce qu'elle ne peut exprimer), de sorte qu'il devient extrêmement difficile de définir différentiellement les valeurs des marqueurs aspectuo-temporels, bien que ceux-ci ne soient pas librement interchangeables en contexte. De plus, la pauvreté de la signification en langue qui leur est reconnue rend pratiquement impossible le calcul de leurs effets de sens en contexte.
\end{abstract}

Nous est-il possible de préciser plus encore cette opposition PR/IMP, décrite vaguement en termes de « différence », de décrochage, de translation, de rupture, d'abandon du repérage par rapport à $<$ Moi, Ici, Maintenant>?

Se contenter d'une telle instruction sémantique pour l'imparfait, dès lors qu'il s'agit de qualifier la valeur en langue, ne constitue pas une dérobade : il s'agit au contraire de prendre acte d'une sous-détermination qui existe dans la langue elle-même. En dire plus serait en dire trop et, automatiquement, privilégier, abusivement, un emploi au détriment des autres, qui sont tout aussi « naturels ».

Si cette vision, au même titre que toutes les approches non-temporalistes, ne devrait pas rencontrer de difficultés insurmontables à traiter les emplois dits modaux, elle devrait affronter l'emploi temporel.

Mais au préalable, un petit rappel concernant les propriétés aspectuelles de l'imparfait, en tout cas dans ses emplois chronologiques, et en dehors de cas de conflit (au sens de Gosselin). Il semble y avoir aujourd'hui unanimité chez les linguistes, quel que soit leur choix théorique et terminologique ${ }^{33}$, pour considérer que l'imparfait marque l'aspect inaccompli, c'est-à-dire, dans le modèle de Gosselin, que " l'intervalle de référence est inclus dans celui du procès, dont les bornes ne sont pas prises en compte » (2010 : 162). Cette propriété aspectuelle est centrale. La vision interne du procès nécessite le déplacement du repère référentiel qui peut être considéré comme un point de focalisation, une fenêtre, à partir desquels le procès est envisagé. Quel autre temps peut offrir cette possibilité de montrer des procès de l'intérieur? Uniquement le présent nous semble-t-il. Mais avec le présent, hors cas de distorsion, il n'y a pas de déplacement de repère référentiel. En bref, si on fait abstraction du présent qui, puisque l'intervalle de référence est couplé à l'intervalle de l'énonciation, ne nécessite pas, dans les cas habituels, un déplacement du repère référentiel pour garantir une lecture aspectuelle de l'inaccompli, l'imparfait est la seule forme temporelle qui doit, pour assurer cette même lecture aspectuelle, décrocher l'intervalle de référence qui doit suivre le procès, de l'intervalle de l'énonciation (auquel il est arrimé par défaut).

Mais où doit-il le suivre?

L'instruction temporelle [+ passé] que la plupart des modèles attribuent à l'imparfait en tant que marqueur n'émerge, selon nous, que contextuellement. Et il semble même que ce n'est pas l'imparfait qui 
en est responsable. Que dit-on, en effet, au juste quand on affirme que l'imparfait localise le procès dans le passé ? Sert-il par lui-même à situer le procès dans le passé ? Est-il simplement compatible avec une localisation du procès dans le passé ?

L'hypothèse défendue ici consiste à retenir la seconde possibilité : c'est parce qu'il est indéterminé, décroché de l'énonciation, qu'un procès à l'imparfait, s'accrochera à des marqueurs chronologiques qui, eux, expriment par eux-mêmes le passé. La valeur d'inaccompli en l'occurrence perturbe plus qu'elle n'éclaire: l'imparfait, ainsi qu'il a été observé dans de nombreux travaux, offre souvent le cadre, l'arrière-plan à des procès au PC (ou au PS). D'où un glissement qui consiste à voir en lui l'élément qui installe le cadre narratif dans le passé, dans lequel se déploient des procès plus ponctuels. On défendra le point de vue contraire : c'est parce qu'il est inaccompli qu'il peut fournir cet arrière-plan, mais ce n'est pas lui le responsable de la localisation dans le passé...

Le cœur du traitement esquissé ici consiste à affirmer que la lecture temporelle de l'imparfait n'est que l'une de ses possibilités interprétatives. Son indétermination référentielle l'oblige à s'attacher aux repères référentiels que le co-texte (PS, PC, adverbiaux de localisation temporelle) ou l'environnement discursif lui attribue. Nous faisons donc l'hypothèse que, dans une situation authentique de communication, il y a toujours un élément co-textuel ou contextuel qui permet l'interprétation chronologique de l'imparfait. Il s'agit d'une hypothèse empiriquement vérifiable : y a-t-il toujours un marqueur qui permette à l'imparfait d'avoir contextuellement une lecture chronologique ? Existe-t-il des cas authentiques où aucun marqueur (y compris contextuel) ne vient donner à l'imparfait cette attache temporelle dans le passé dans lequel il parviendrait tout de même à situer le procès ?

Plus généralement, la démarche ne peut être validée et la valeur retenue que si on parvient à décrire à grande échelle les différents emplois et prédire leurs sens en contexte. Pour cela, le modèle devrait s'appuyer sur l'étude d'un vaste corpus de données authentiques.

\section{En guise de conclusion}

Sur la base de considérations morphologiques, l'hypothèse défendue ici consiste à scinder la catégorie traditionnelle « temps » en deux sous-catégories distinctes :

une première catégorie (Y) qui fixe le repère énonciatif à partir duquel se déploie la temporalité des procès. Cette catégorie énonciative permet d'opposer deux groupes de Formes Temporelles, selon le marqueur choisi (Ø ou /i/).

1. Hors distorsion, le premier groupe (qui a pour centre le présent) est celui de l'assertion dans la sphère <Moi-Ici-Maintenant $>$ et inclut, en plus du PR, le PS, le FS et leurs formes composées ou périphrastiques correspondantes. Ces formes s'opposent, mais à l'intérieur d'un même paradigme énonciatif, par l'instruction temporelle (et éventuellement aspectuelle) que chacune d'entre elles code. Ici, aussi, on suivra Damourette \& Pichon qui parlent de localisation temporelle "sèche" particulièrement visible au PS. On ne développera pas davantage la vision aspectuelle qu'un tel système ne manquera pas d'impliquer: on pense qu'il est possible de se passer de toute caractérisation aspectuelle pour décrire le PS. Il ne serait dans cette vision qu'une forme purement temporelle. En cela le traitement proposé ici serait sans doute plus guillaumien que ceux qu'on rencontre chez les disciples de Guillaume, dont «[b]eaucoup [...], ainsi que le constate Wilmet (1997: 332), n'ont pas accepté sans états d'âme qu'il rejette en dehors du domaine ne fût-ce que le couple marchai/marchais ». L'une des principales motivations qui a poussé à traduire ce qui n'était chez Guillaume qu'une caractérisation négative (le global par opposition au sécant, seul porteur parmi les deux formes d'une valeur aspectuelle) en une caractérisation positive est le maintien au sein du système d'un rapport différentiel au sein de ce couple. Dans notre vision, il ne s'agirait pas d'un couple, leurs différences se situant ailleurs, ils n'auraient pas besoin de s'opposer aspectuellement pour maintenir la « symétrie » du système. 
2. Le second groupe a pour centre l'imparfait et contient également le conditionnel et leurs formes composées. Il s'agit de formes temporelles a priori indéterminées parce qu'elles codent des instructions aspectuo-temporelles calculées par rapport à un intervalle de référence lui-même a priori indéterminé. Leur repère référentiel se définissant en langue uniquement par sa différence (décrochage) par rapport à l'intervalle d'énonciation, ces formes sont susceptibles de prendre toutes sortes de significations contextuelles (aussi bien énonciatives que chronologiques). On comprendra ainsi pourquoi le phénomène de la polysémie temporelle, s'il est général, s'observe surtout avec ces formes temporelles. On comprendra aussi pourquoi, contrairement à la localisation sèche du PS (où le procès passé est vu du présent), l'imparfait, lorsqu'il parvient à localiser contextuellement dans le passé, donne cet effet de sens « autre époque ${ }^{34}$ » (c'était mieux avant, c'était une autre époque)...

- $\quad$ une seconde catégorie $(\mathrm{X})$, que l'on peut qualifier d'aspectuo-temporelle, et qui donne à la forme temporelle ses caractéristiques temporelles (présent, passé, futur, ou, mieux encore, si on exprime le phénomène en termes de relations temporelles entre le procès et le repère référentiel, coïncidence, antériorité et postériorité) et, éventuellement, aspectuelle (essentiellement l'accompli, pour les formes composées, et l'inaccompli pour le PR et l'IMP). Nous retrouvons là les relations temporelles habituelles qui s'opèrent entre les repères référentiels et les procès.

Cette vision schématique (car elle laisse ouvertes différentes possibilités) semble offrir de nombreux avantages, et sans doute présente-t-elle aussi des difficultés qu'on n'entrevoit pas encore. Parmi les avantages visibles, on peut évoquer ici son économie : elle met en place moins de catégories : non seulement, elle abandonne la catégorie de l'aspect global puisque la simple localisation dans le temps suffit à décrire le fonctionnement des formes concernées, mais en plus elle ne propose le recours à un intervalle de référence autre que $\mathrm{S}$ (le fameux point référentiel $\mathrm{R}$ de Reichenbach) que s'il y a décrochage par rapport à la situation d'énonciation. Autrement dit, pour le premier groupe de formes temporelles (où $\mathrm{R}=\mathrm{S}$ ), il est proposé de calculer la relation temporelle du procès directement par rapport à $\mathrm{S}$, ce qui est en cohérence avec la vision globale esquissée ci-dessus. En plus de son économie, cette vision semble garantir une cohérence sur un point difficile, nous l'avons déjà vu au niveau morphologique, et auquel la plupart des modèles ont dû apporter une réponse qu'on peut juger ad hoc en proposant un repère spécifique pour le conditionnel : ici, le conditionnel se déploie à partir du même repère référentiel que l'imparfait.

Les différentes hypothèses esquissées ici peuvent trouver dans le modèle de la Morphologie Distribuée (Halle et Marantz 1993 \& 1994) un cadre de traitement naturel. Disposant d'un composant morphologique autonome «chargé de convertir les structures motivées syntaxiquement et sémantiquement en structures satisfaisant des exigences indépendantes de la phonologie » (Harris, 1997 : 35), la MD est logiquement compatible avec n'importe quel modèle grammatical, dès lors qu'il aura clairement établi l'input du Composant Morphologique. Les morphèmes y sont vus comme des faisceaux de traits sémantiques, syntaxiques et morphologiques abstraits, sans réalisation phonologique. Celle-ci n'est fournie qu'après les opérations morphologiques (insertion tardive, qui intervient après les règles morphologiques de fusion, fission, appauvrissement...).

\section{Références bibliographiques}

ABOUDA, L. (1997). Le conditionnel : temps ou mode ? Arguments syntaxiques, Revue Romane, 32, 2, 179-198.

ABOUDA, L. (2001). Les emplois journalistique, polémique, et atténuatif du conditionnel. Un traitement unitaire, in P. DENDALE et L. TASMOWSKI (eds), Le conditionnel en français, Metz, Université de Metz.

ABOUDA, L. (2015). Syntaxe et Sémantique en corpus. Du temps et de la modalité en français oral, Habilitation à Diriger des Recherches, Université d'Orléans.

ARNAULD, A. \& LANCELOT, Cl. (1660, éd. 1972). Grammaire générale et raisonnée de Port-Royal, Paris, Slatkine. 
ARONOFF, M. (2012). Morphological stems: what William of Ockham really said, in Word Structure 5.1, p. $28-51$.

BARCELÓ, G. \& BRES, J. (2006). Les temps de l'indicatif en français, Paris, Ophrys.

BONAMI, O. (2014). La structure fine des paradigmes de flexion. Études de morphologie descriptive, théorique et formelle, mémoire HDR, Université Paris 7 Denis Diderot.

BONAMI, O. \& BOYE, G. (2003). Supplétion et classes flexionnelles dans la conjugaison du français, Langages 152, $102-126$.

BONAMI, O. \& BOYE, G. (2007). Remarques sur les bases de la conjugaison, in d'Elisabeth Delais-Roussarie et Laurence Labrune (dir.), Des sons et des sens, Paris, Hermès, 77-90.

BONAMI, O. \& BOYE, G. (2010). La morphologie flexionnelle est-elle une fonction ?, in Injoo Choi-Jonin, Marc Duval et Olivier Soutet (dir.), Typologie et comparatisme, hommage offert à Alain Lemaréchal., Leuven : Peeters, 21-35.

BONAMI, O. \& BOYE, G. (2014). De formes en thèmes, in Florence Villoing, Sarah Leroy et Sophie David (dir.), Foisonnements morphologiques. Etudes en hommage à Françoise Kerleroux, Presses Universitaires de Paris Ouest, $19-44$.

BOYÉ, G. \& CABREDO HOFHERR, P. (2006). The structure of allomorphy in Spanish verbal inflection, Cuadernos de Lingüística, t. 13. Instituto Universitario Ortega, y Gasset, 9-24.

BRUNOT, F. (1905). Histoire de la langue française des origines à nos jours, éd. 1966, Paris, Armand Colin.

BRUNOT, F. (1922). La Pensée et la langue, Paris, Masson et Cie.

CAMUSSI-NI, M.-A. (2006). Analyse formelle et conceptuelle des formes verbales du français contemporain : A la croisée du passé simple et de l'imparfait, du futur et du conditionnel, les concepts « \pm potentiel » et « défini », Thèse de l'Université Rennes 2.

CAUDAL, P. \& VETTERS, C. (2005). Un traitement conjoint du conditionnel, du futur et de l'imparfait : les temps comme des fonctions d'acte de langage, Cahiers Chronos, 12, Temporalité et attitude, 109-124.

CLARIS, J.-M. (1971). Notes sur les formes en -rait, Langue française, 11, 32-38.

COMRIE, B. (1985). Tense, Cambridge University Press.

CONFAIS, J.-P. (1990). Temps, Mode, Aspect, Toulouse, Presses Universitaires de Mirail.

COSERIU, E. (1980). Aspect verbal ou aspects verbaux ? Quelques questions de théorie et de méthode, in J. David \& R. Martin (dir.), La Notion d'aspect. Actes du colloque de Metz. Paris, Klincksieck.

CULIOLI, A. (1980). Valeurs aspectuelles et opérations énonciatives: l'Aoristique, in J. David \& R. Martin (dir.), La Notion d'aspect. Actes du colloque de Metz. Paris, Klincksieck, 181-193.

CURAT, H. (1991). Morphologie verbale et référence temporelle en français moderne. Essai de sémantique grammaticale, Genève - Paris, Droz.

DAMOURETTE, J. \& PICHON, E. (1911-1927). Des Mots à la pensée : essai de grammaire de la langue française, Paris, D'Artrey, 1970.

DE MULDER, W. (2012). Un sens épistémique pour l'imparfait et le passé simple ?, Langue française, 173, 99-113.

DE SAUSSURE, L \& STHIOUL, B. (2005). Imparfait et enrichissement pragmatique, Cahiers Chronos, 14, $103-210$.

FEUILLET, J. (1988). Problématique de l'auxiliation, in Cahiers du CERLICO, La Question de l'auxiliaire, 1-38.

FOURNIER, N. (1986). L'imparfait chez les grammairiens classiques, de Maupas (1607) à Restaut (1730), in P. Le Goffic (1986, éd.), 13-41.

FUCHS, C. (1986). L'ambiguïté et la paraphrase en psycho-mécanique : l'exemple de l'imparfait, in P. Le Goffic (1986, éd.), 43-54.

GIRARD, Abbé (1747). Les vrais principes de la langue françoise, éd. de 1982, Genève, Paris, Droz.

GLEASON, (1969). Introduction à la linguistique, Larousse.

GOSSELIN, L. (1996). Sémantique de la temporalité en français. Un modèle calculatoire et cognitif du temps et de l'aspect, Louvain-la-Neuve, Duculot, col. « Champs linguistiques ». 
GOSSELIN, L. (2001). Relations temporelles et modales dans le "conditionnel journalistique". In Patrick DENDALE et Liliane TASMOWSKI (eds), Le conditionnel en français, Metz, Université de Metz.

GOSSELIN, L. (2005). Temporalité et modalité, Bruxelles, De Boeck-Duculot, col. « Champs linguistiques ».

GOSSELIN, L. (2010), La validation des représentations. Les modalités en français, Amsterdam-New York, Rodopi, coll. Etudes Chronos.

GUILLAUME, G. (1929). Temps et verbe. Paris, Honoré Champion. [Réimprimé en 1965].

HAILLET, P.P. (2002). Le conditionnel en français : une approche polyphonique, Gap, Ophrys.

HAILLET, P.P (2003). Représentations discursives, point(s) de vue et signifié unique du conditionnel, Langue Française, 138, 35-47.

HAILLET, P. (2007). Pour une linguistique des représentations discursives, Bruxelles, Duculot-De Boeck.

HALLE, M. \& MARANTZ, A. (1993). Distributed morphology and the pieces of inflection, MIT Working Papers in Linguistics, Vol. 21. Ed. by Carnie \& Harley, 275-288

HALLE, M. \& MARANTZ, A. (1994). Some key features of Distributed Morphology, in MITWPL 21: Papers on phonology and morphology, ed. Andrew Carnie and Heidi Harley, MITWPL, Cambridge, 275-288.

HARRIS, J. (1997). Morphologie autonome et pronoms clitiques en catalan et en espagnol, in Zribi-Hertz, A. (ed.), Les Pronoms, Paris 8, Presses Universitaires de Vincennes.

HISQUIN, D. (2000). « Au conditionnel », Les Revues pédagogiques de la Mission Laïque Françaises, 38, 13-23.

HUOT, H. (2001). La morphologie. Forme et sens des mots du français, $2^{\mathrm{e}}$ édition, revue et actualisée, Armand Colin, col. Cursus.

IMBS, P. (1960). L'Emploi des temps verbaux en français moderne, Paris, Klincksieck.

ISAAC, L. (1985). Calcul de la flexion verbale. Genève, Droz.

KLEIBER, G. (1993). Lorsque l'anaphore se lie aux temps grammaticaux, in Vetters (éd.), Le Temps, de la phrase au texte. Lille, PUL, 117-166.

LEBAUD, D. (1993). IMP : indétermination aspectuo-temporelle et changement de repère, Le Gré des langues, 5, 160176.

LE GOFFIC, P. (éd.). (1986). Points de vue sur l'imparfait, Caen, Centre de Publications de l'Université de Caen.

LE GOFFIC P. (1995). La double incomplétude de l'imparfait, Modèles linguistiques, 31, 133-149.

LE GOFFIC, P. (1997). Les formes conjuguées du verbe français, oral et écrit, Paris, Ophrys.

MAINGUENEAU, D. (1981). Approche de l'énonciation en linguistique française, Paris, Hachette.

MOLENDIJK, A. (1993). Présuppositions, implications, structure temporelle, in C. Vetters (éd.), Le Temps, de la phrase au texte. Lille, PUL, 167-191.

MOLENDIJK, A. (1996). Anaphore et imparfait : la référence globale à des situations présupposées ou impliquées, Cahiers Chronos, 1, Anaphores temporelles et (in)cohérence, 109-124.

MONVILLE-BURSTON, M. \& BURSTON, J. (2005). Retour à « remise de peine » : l'imparfait, un toncal à faible marquage, Cahiers Chronos, 14, 135-156.

MORIN, Y.-Ch. (1987). Remarques sur l'organisation de la flexion en français, ITL Review of Applied Linguistics 77-78, $13-91$.

PATARD, A. (2007). L'un et le multiple. L'imparfait de l'indicatif en français : valeur en langue et usages en discours, Thèse de l'université Paul-Valéry, Montpellier III.

PICABIA, L. (1999). Morphologie autonome et morphologie verbale du français : une représentation de l'auxiliaire, Langages, 135, 46-62.

PINCHON, J. \& COUTE, B. (1981). Le Système verbal français. Paris, Nathan. 
PLENAT, M. (1987). Morphologie du passé simple et du passé composé des verbes de 1' "autre" Conjugaison, ITL Review of Applied Linguistics 77-78, 93-150.

RIEGEL, M., PELLAT, J.-C. et RIOUL, R. (1994). Grammaire méthodique du français, Paris, PUF.

SABANEEVA, M. (1993). Mode verbal et problèmes connexes. Essai d'une formule nouvelle, La Linguistique, 29, 1, 5565.

STUMP, G. T. (2001). Inflectional Morphology. A Theory of Paradigm Structure. Cambridge, Cambridge University Press.

TASMOWSKI-DE RYCK, L. (1985). L'Imparfait avec et sans rupture. Langue française, 67, 59-77.

TOURATIER, C. (1996). Le système verbal français (Description morphologique et morphématique), Paris, Masson \& Armand Colin, Col. U.

VET, C. (1996). Anaphore et deixis dans le domaine temporel. Cahiers Chronos, 1, Anaphores temporelles et (in) cohérence, 147-163.

VETTERS, C. (1996). Temps, aspect et narration, Amsterdam, Atlanta, Rodopi.

WAGNER, R.-L. (1939). Les Phrases hypothétiques introduites par si dans la langue française des origines à la fin du XVIe siècle, Paris, Droz.

WAGNER, R.-L. \& PINCHON, J. (1962). Grammaire du français classique et moderne, Paris, Hachette.

WILMET, M. (1996). L'imparfait : le temps des anaphores ?, Cahiers Chronos, 1, Anaphores temporelles et (in)cohérence, 199-215.

WILMET, M. (1997). Grammaire critique du français, Louvain-la-Neuve, Duculot.

YVON, H. (1952). Faut-il distinguer deux conditionnels dans le verbe français ? Le Français moderne 4 (20è année).

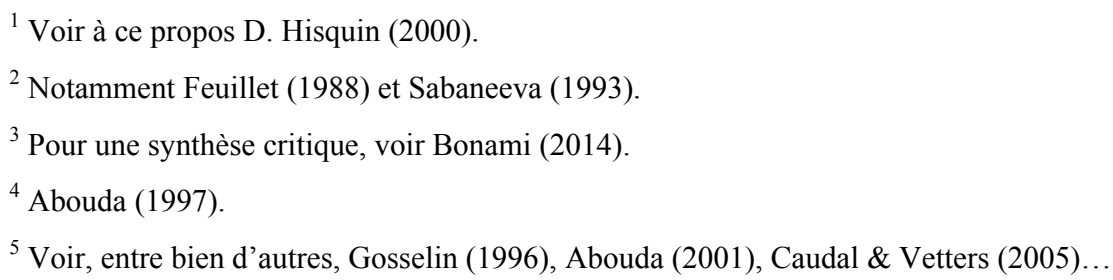
(1991)..., les travaux ayant abordé cette question, plus ou moins longuement, sont nombreux. Voir, pour un bon résumé et une mise en perspective, Camussi-Ni (2006). Le débat se poursuit entre Boyé \& Cabredo Hofherr (2006) et Spencer (2012), et engage jusqu'à l'identification de l'affixe et le radical verbal...

${ }^{7}$ Voir, pour s'en rendre compte, Aronoff (2012).

${ }^{8}$ Voir Picabia (1999) pour une proposition d'intégration de ces flexions dans le cadre de la morphologie distribuée et du programme minimaliste.

${ }^{9}$ Personne-Nombre

${ }^{10}$ Le principe est suivi par Pinchon \& Couté (1981) qui font disparaitre le zéro, qu'ils avaient admis dans leur description du futur et du conditionnel, pour le remplacer dans les tableaux par des cases grisées, et en généralisant le système de la sorte (1981: 41) : «Entre la base et la désinence peut s'insérer un élément ou deux éléments ».

${ }^{11}$ L'abandon de l'hypothèse de la compositionnalité du conditionnel ne remet pas automatiquement en cause son appartenance à l'indicatif, si on parvient par ailleurs à la justifier. On pourrait en effet appréhender la forme en -rait comme une marque indécomposable, support formel d'un seul et unique morphème, faisant partie d'un paradigme où il s'oppose aux divers autres temps de l'indicatif, y compris le futur et l'imparfait. Comme le souligne Touratier (1996 : 61), «en vertu du caractère arbitraire du signe linguistique, la langue peut fort bien utiliser la même unité morphologique comme signifiant de deux morphèmes différents, que l'on qualifie alors d'homonymes ». Cela signifie concrètement que la marque $-r$ - qui existe dans le morphème du conditionnel pourrait être considérée comme distincte du morphème $-r$ - du futur... 
${ }^{12}$ D'autant que les deux formes entretiennent des relations sémantiques indéniables, ainsi que le rappellent Wagner et Pinchon (1962 : 303) : "Il est vrai que le futur et le conditionnel évoquant l'avenir se chargent parfois de plus de valeurs affectives que les temps relatifs au présent ou au passé. C'est pour cette raison que les grammairiens qui considèrent les modes comme des formes de la sensibilité ont été conduits à traiter du conditionnel comme d'un mode. Pour être conséquents, ces grammairiens devraient aussi considérer le futur comme un mode. »

${ }^{13}$ L'auteur reconnait $(1951$ : 271) que l'idée d'opposer le 'mode' futur à l'indicatif remonte au moins à Destutt de Tracy (1803) qui opposait les formes de l'existence positive aux formes de l'existence éventuelle (futur, conditionnel, simples et composés).

${ }^{14}$ Se reposera alors la question de savoir si ce - $R$ - est différent de celui de l'infinitif...

${ }^{15}$ Voir Pinchon \& Couté (1981), et la note 10.

${ }^{16}$ C'est la seule forme temporelle simple de l'indicatif qui a besoin, dans le système de Gosselin par exemple, de deux intervalles de référence distincts... Il le reconnaît explicitement lui-même (2001: 57) : "Cette exigence est tout à fait singulière dans le système verbal du français. Elle peut être mise en rapport avec la constitution morphologique du conditionnel, qui articule deux morphèmes temporels (l'un qui exprime le passé, et l'autre la postériorité). »

${ }^{17}$ Voir par exemple le traitement de Camussi-Ni (2006), très différent de celui qui sera proposé ici, malgré des choix méthodologiques initiaux comparables.

${ }^{18}$ Il s'agit non pas d'une opposition entre les formes verbales simples et complexes, telle qu'on se la figure actuellement, mais bien d'une opposition notionnelle équivalente à celle qui m'occupe ici, puisque les auteurs distinguent nettement entre les temps « considérés simplement dans leur nature de présent, de prétérit et de futur » et les « temps composés dans le sens » (1660:76) parmi lesquels figure l'imparfait.

${ }^{19}$ Voir aussi encore récemment Vet (2005). Tout cela sans parler du modèle de Benveniste et celui de Weinrich, dont la bipartition en plans ne correspond que partiellement au domaine temporel.

${ }^{20}$ Voir Vetters (1996), pour une présentation historique assez détaillée mais qui, curieusement, ignore Damourette \& Pichon.

${ }^{21}$ Une linguistique générale, mais pas dans une perspective typologique... C'est pour rendre compte, dans le système de Reichenbach, de certaines formes temporelles de l'anglais, i.e. le " pluperfect » et le " future perfect », qu'il a été amené à reconnaître la classe du « combined absolute-relative tense » $(1985: 125$ sq.).

22 « La conclusion que nous tirerons de cet aperçu de l'indicatif, écrit Fournier (1986: 19), est que l'imparfait, de par son appartenance sans discussion à ce mode, est voué à l'expression du certain et de l'assertion pleine, ce qui ne laisse pas place a priori aux emplois dits modaux de ce tiroir qui sont renvoyés au subjonctif latin, sans autre commentaire ». Le glissement Certain > Réel > Passé fera le reste. Voir Gosselin (2010 : 73 sq.).

${ }^{23}$ Plus précisément, pour ces auteurs, la lecture temporelle de l'IMP n'est que l'une de ses lectures possibles qui ne semble émerger que compositionnellement: «il ne semble pas douteux que le rôle du saviez comme agent d'expression d'un passé n'est qu'un cas particulier de son sens toncal » (§ 1707).

${ }^{24} \mathrm{Ou}$ encore, l’IMP décrit « un monde de phénomènes centrés autour de quelque chose d'autre que le moi-icimaintenant » (§ $1740: 234)$.

${ }^{25}$ On peut aussi se reporter par exemple à Le Goffic (1986), et plus généralement, à tous les articles rassemblés dans son recueil, ou encore, plus récemment, à Monville-Burston \& Burston (2005)...

${ }^{26}$ Monville-Burston \& Burston notent (2005: 135) à juste titre : « Ce qui singularise l'imparfait [...] c'est la multiplicité de ses usages. Pour avoir une idée de cette diversité, il suffit de se reporter aux différentes rubriques sous lesquelles l'imparfait est décrit dans le classique Bon Usage : quinze, comparées à seulement quatre pour le passé composé et tout juste deux pour le passé simple; de tous les autres temps, c'est le présent qui arrive bon second avec les six usages que M. Grevisse lui reconnaît».

${ }^{27}$ Voir les observations de C. Fuchs (1986, notamment 51 sq.), qui, discutant du traitement psychomécanique de l'imparfait, souligne une certaine hésitation du modèle vis-à-vis du rôle attribué au contexte. Il semblerait qu'il faille lui reconnaître un plus grand rôle puisqu'il semble en grande partie responsable des coupes dans les saisies... Ce qui invaliderait le raisonnement discuté ici. 


\footnotetext{
${ }^{28}$ Identifié quelquefois comme un emploi à part : imparfait exprimant un souhait ou un désir...

${ }^{29}$ Les emplois (a) et (b) sont souvent rangés dans les études les plus récentes dans une classe distincte sous le chapeau du contrefactuel. Mais cette même classe est rangée par Patard (2007) dans la classe temporelle...

${ }^{30}$ Les mécanismes descriptifs et explicatifs peuvent être (et quelquefois sont) investis pour traiter des phénomènes qui ne relèvent pas de la temporalité.

${ }^{31}$ Qui attribue au marqueur une valeur première en langue et des sens secondaires et dérivés en contexte.

32 Au-delà de l'inadéquation théorique de ces démarches, Gosselin a montré (2010: 109 sq.) qu'elles sont inadéquates sur le plan empirique : en neutralisant (par effacement des traits sémantiques) certaines propriétés du marqueur, on perd des éléments nécessaires à l'interprétation globale de l'énoncé. Voir également Confais (1990).

${ }^{33}$ Vision sécante (Guillaume), vision interne (Martin), aspect sécant (Wilmet), imperfectif, etc. : on parle de la même chose, ou presque.

${ }^{34}$ Le Il était une fois ne localise pas dans le passé (malgré les formules trompeuses qui peuvent suivre l'expression comme il y a très très longtemps) mais ouvre un univers différent, magique, celui du conte... Mais, on le sait, ne peut être raconté que ce qui se donne pour passé.
} 